\title{
IoT for health-based system: analog network coding with energy harvesting
}

\author{
Dalal Abdulmohsin Hammood ${ }^{1}$, Mahmoud Shuker Mahmoud ${ }^{2}$, Ahmed Alkhayyat ${ }^{3}$ \\ ${ }^{1}$ Electrical Engineering Technical College-Department of Computer Technical Engineering, \\ Middle Technical University (MTU), Iraq \\ ${ }^{1}$ Bioelectromagnetics Research Group (BioEM), School of Computer and Communication Engineering, \\ Universiti Malaysia Perlis (UniMAP), Malaysia \\ ${ }^{2}$ Department of Computer Technical Engineering, Al-Mansour University College, Iraq \\ ${ }^{3}$ Department of Computer Technical Engineering, College of Technical Engineering, The Islamic University, Iraq
}

\begin{tabular}{|c|c|}
\hline Article Info & ABSTRACT \\
\hline Article history: & In the recent health-care field, the Interent of health thigs (IoHT) \\
\hline Received Feb 18, 2020 & $\begin{array}{l}\text { technologies provide appropriateness between doctors and people receiving } \\
\text { medical treatment, because they are useful to several curative domains. }\end{array}$ \\
\hline Revised Apr 16, 2020 & The wireless body sensors networks which can be abbrivated as every \\
\hline Accepted May 1, 2020 & $\begin{array}{l}\text { technology of a WBSN is an important one from IoT growing's of } \\
\text { the health-care model, as all sick people are observed utilizing some set of }\end{array}$ \\
\hline Keywords: & $\begin{array}{l}\text { nodes of sensors that are light as well as with no high power. In this paper, } \\
\text { we proposed Energy Harvesting-based Inter-WBSN cooperation Network }\end{array}$ \\
\hline Analog network coding & Coding (EH-IWCNC) for Internet of Medical Things. \\
\hline
\end{tabular}

IoT

Outage probability

\begin{abstract} technologies provide appropriateness between doctors and people receiving medical treatment, because they are useful to several curative domains. the health-care model, as all sick people are observed utilizing some set of nodes of sensors that are light as well as with no high power. In this paper, Coding (EH-IWCNC) for Internet of Medical Things.
\end{abstract}

\section{Corresponding Author:}

Dalal Abdulmohsin Hammood,

Electrical Engineering Technical College,

Department of Computer Technical Engineering,

Middle Technical University (MTU), Al Doura 10022, Baghdad, Iraq.

Email: dalal.alsaady@eetc.mtu.edu.iq \& dalalmmf59@gmail.com

\section{INTRODUCTION}

The IoT has been a developing eco-system which merges durable equipment, gadgets, materialistic things, software, as well as organisms which include humans and other creatures on some networking that makes them able to react, connect, gather as well as reciprocate information, and there are different major types of IoT services, from the industrial applications to eHealth care applications. In this work, the smart wearable/implants sensors, machine devices attached on or inside a human body for monitoring in a hospital digital healthcare system are concerned, for collecting information concerning the wellness and illness relative level status of a person for the pulsation of the heart, the pressure of the blood, blood sugar level, etc., throughout sensors on the fashion electronics [1-4].

The technologies of WBSN can be considered as a technology that is very imperious and has got the ability to be utilized within IoT-based contemporary maintenance of health pattern [5]. The technology of WBSN can be considered as a set of appliances that are light and of lower power, accompanied by a transceiving device that utilized for monitoring the essential indications come off people. All of the WBSN's sensors are able of gathering mental indications like EEG and ECG, besides BT as well as movement and forwards the gathered indications towards coordinator node which can be abbrivated as $\mathrm{CN}$ on a medium carried by radio or microwave frequencies, or by HBC, that is human body channel to be analyzed more as well as inspecting [6]. The patterns of WBSN have the capability to give extended term health-care observing of human and there will not be any need of 
limiting their capability of moving or the efficacy. Those patterns can be utilized for creating a smart and cheap health-care monitoring and it is utilized for the diagnostic procedure [7].

Although its widespread development, the IoT will be within the primitive phase of its and possesses a large space to look for within various topics such as standards, the system's characteristic for dealing with an increasing quantity in achieving a work via supplying the system with more than one source, diverseness of constituent structure, joint definition language, service of specific field detection, integrating accompanied by standing systems of Information Technology, etc. Within the written works, some works in the patterns of IoT are existed, that established on health, for instance, safe IoT paradigms established on maintenance of health operate on WBSN is presented in [8]. Several recent studies in WBSN area are reviewed in [9] accompanied by a certain concentration on the consuming that its power is not high, transmitting accuracy, the time interval between the stimulation and response, the transmission speed or the number of bits per second transferred, and secureness. In addition to this, the authors considered the needs as well as the WBSN requirements in a conventional e-healthcare frame-work which can discover how such systems are able of having efficient communication in the home environment network. A tied mathematical expression presented and studies in [10] for the built-in and built-out channel statistical model that could describe the indication travelling among transmitter and receiver antennas.

The analysis is built on the three-dimentional immitating model of a body. In [11], the authors took into account a novel design for the Internet of Things health maintenance patterns that is pointed out as a safe electronic maintenance of health pattern established on Internet of Things using BSN-care. The end-to-end secureness scheme has been presented in [12] for the dynamic maintenance of health Internet of Things patterns. The analyzing shows that the scholars utilized the conception of fog layers in Internet of Things to realize smooth ability of moving for the fog expanding the system of cloud towards the network's verge. the Internet of Things' in the ehealthcare industries is studied as well as presented in [13], that identifies the intelligentizing tendency for upcoming study about ehealthcare patterns built on Internet of Things. The on body sensors with harvesting energy capabilities based on the heat from the Sun is studies and modelled in [14]. Along with this, some mobile app that can be accessed over a network has been designed to show the collected information off a body.

The effect of the power allocation and packet size determination is presented in [15] for the wireless environment, over the maintenance of heath's performing which established on Internet of Things system are considered and investigated. In the study, three different protocols are proposed: decision of power scale, decision of power size and scale, decision of international linking. A novel Internet of Things aware Smart Hospital System is designed and presented in [16], the proposed design system is able to appropriately manage the emergency situations. Interoperability stayed a big load for the researchers as well as Internet of Things system improvers. But in [17], a model that can exchange data with unambiguous, shared meaning established on Internet of Things is proposed that is given a semantic interoperability for the heterogeneous IoT device. Doctors can get hold of the sick people of theirs because the gathered data transmitted among one another were explained and communicated in a semantic rationally. In [18], a developed conception is set up between cloud computing and Internet of Things: the cloud Internet of Things health (CC-IoT) systems.

This CC-IoT as well as numerous key integrating issues can be investigated for demonstrating a functional seeing which integrated present mechanism of CC and IoT in apps of maintenance of health. A protector of privacy has been suggested in [19], that provides protection for the information privacy gathered from a sick person. As the Slepian-Wolf sharing of secrets established on coding can be utilized within Privacy Protector overcoming several kinds of securing mechanisms. A second kind of fuzzy assisted by ontology recommending regulation is given in [20] to maintenance of health established on Internet of Things for skillfully observing sick people's bodies. This system greatly improved the predicting accurateness of sick people's situation as well as accuracy rate for medical as well as the nutrition suggestions. An energy harvesting IoT pattern established on health in [21] to period of out of power possibility reducing throughout the network cooperation of inter-wireless body area has been introduced.

The contributions of this paper are summarized as follow: A pattern of maintenance of health established on Internet of Things can be made and proposed that depicts information travelling over different tiers. In addition, inter-WBSN cooperation integrated with energy harvesting is proposed, the proposed protocol is named as EHIWCNC or Energy Harvesting-based Inter-WBSN cooperation Network Coding for Internet of Medical Things. Finally, an explicit mathematical term for the possibility of out of work period can be originated.

What remains of the paper can be arranged just like the following: The architecture of WBSN can be depicted with the second section, that includes a couple of small sections in its turn, a WBSN within a network of health established on Internet of Things, as well as novel corporation of inter WBSN besides the essential process belongs to suggested protocols. And in the third section, the connection analysis and possibility of out of work period for direct transmission as well as suggested protocol can be looked at. The energy competence for various transmitting scenarios as well as suggested protocols are investigated in Section IV. The performing as well as outcomes can be presented within the fifth section. In the end, future work and conclusion can be drawn within the sixth section. 


\section{WBSN NETWORKS ARCHITECTURE}

\subsection{WBSN in IoT-based Health Network}

WBSN in a Network of health established on Internet of Things We can see a novel architecture of a pattern of Internet of things established on health within the Figure 1 that is parted into 4 layers, where, all layers belong to the suggested architecture explained in the way mentioned below:

a) Layer of WBSN, layer\#1, within the layer, the sensors could be connected in a direct way to a body of a human or sewed into material (the sensor that can be worn) or instilled in a body of a person. Sensors like those could be EMG, ECG, or EEG etc... The information booked by the sensors can be transferred towards $\mathrm{CN}$ by over the air 802.15.6 standard, then $\mathrm{CN}$ transfers the things that have been transferred via sensors towards the following tier over one of the wires of over the air technology.

b) Smart Interface, layer\#2, within the layer, smart devices can be used, (that is to say, mobile or PC). In the layer here, information have been investigated and analyzed, after that transmitted towards layer 3 on a chosen over the air communicating technology (In another word BT, cellular Base station, Wi$\mathrm{Fi}$ ). The second layer represent a bridge tier that is the link of a WBSN and the is the materialistic machinery, transmitting media, as well as programs utilized in connecting PCs and people who use them to the Net, as well as some time placed in an area of WBSNs.

c) Infrastructure Net layer, layer\#3, the level bridge a gap among layer\#2 and layer\#4 by going out of communicating science.

d) Services related to cars layer (layer\#4), in the layer here, the received information patient server, the Patient server: in this layer, the data are stored, analyzed and sent towards the proper service like the emergent section, doctor, or relatives.

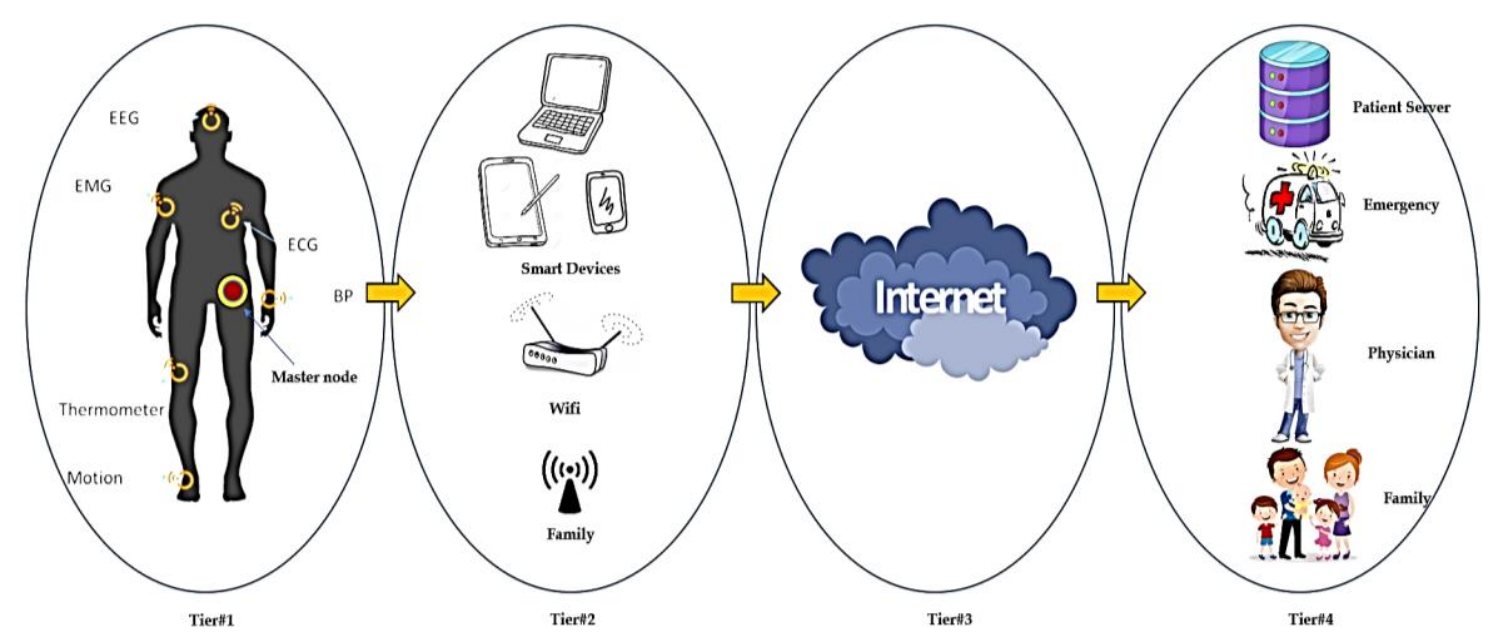

Figure 1. WBSN in IoT-based health networks

\section{ENERGY HARVESTING-BASED OVER INTER-WBSN COOPERATIVE NETWORK CODING}

In the conventional topology of WBSN networks, various sensors distributes in an identical way on a body for monitoring every significant sign, after that, the sensor gathered and transfers information towards the $\mathrm{CN}$ [22]. Hence, WBSNs are built on the topology of single hop star, the entire sensors tranfers the collected information on over the air environment or $\mathrm{HBC}$ towards the $\mathrm{CN}$ [23]. The $\mathrm{CN}$, after that forwards the information towards the following tier like clarified before.

In the near future, the WBANs are possibly overlap and communicate with each other, and this make the cooperation between WBAN sensors is possible (cooperation coomunication method is most suitable method for WBAN because it is considered plug and play method [24-28]). Then, we provided new design, which allow the on body nodes cooperate with each other, named as inter-WBAN cooperation. On other hand, involving nodes in cooperation which mean serving the other node transmission could signaficantly effect the battery life and cosnume channel resiurces because the relay need to transmit at two different time slots, one slot for first source and second slot for the second source, thus in this paper the mn apply network coding over two received signle [29, 30]. In addition, along with cooperation aware network coding, we assumed the layer\#2 devices transmit energy over radio signal to collaborated nodes as it shown in the Figure 2. 


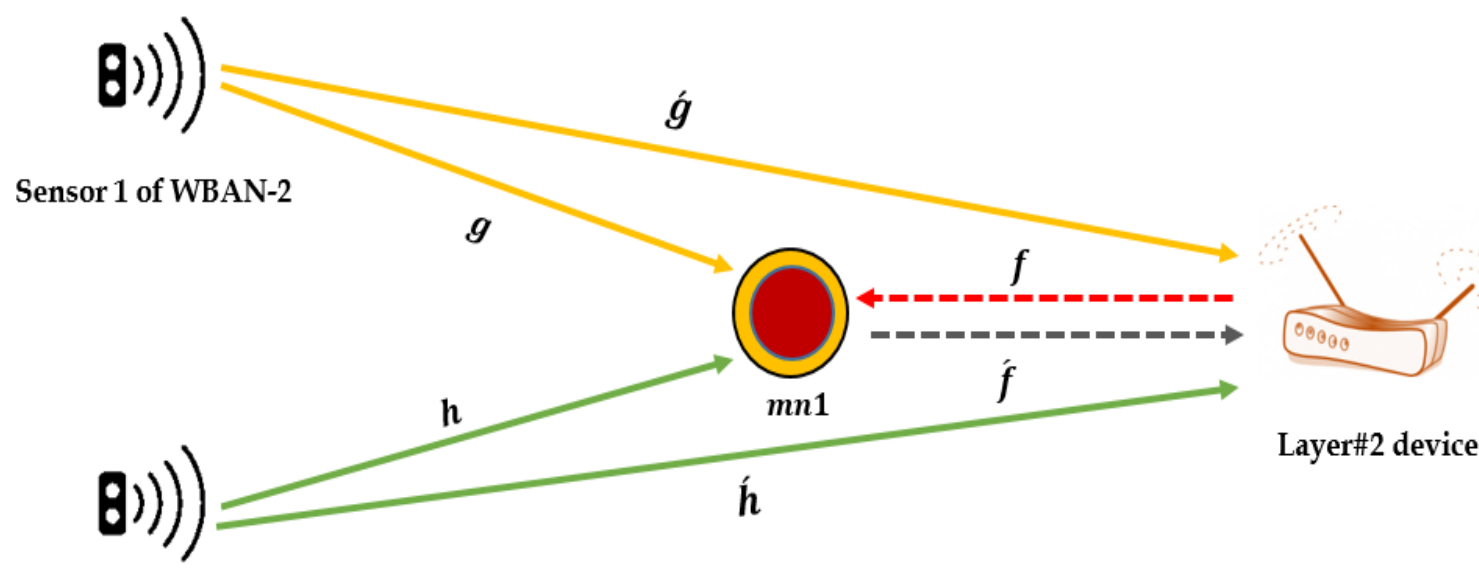

\section{Sensor 1 of WBAN-1}

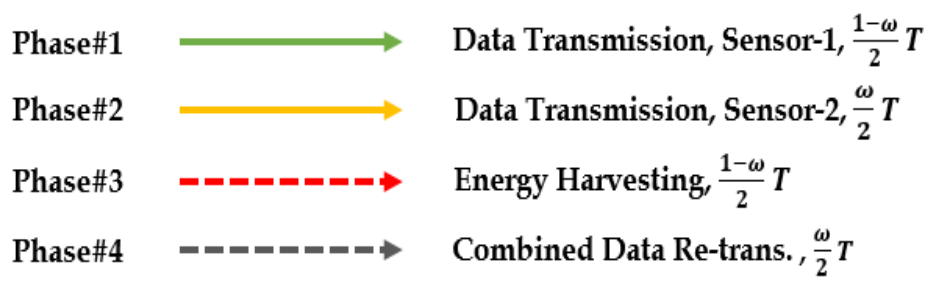

Figure 2. Energy harvesting over inter-WBSN analog network coding which can be abbrivated as (EH-IWANC) architecture

\section{OUTAGE PROBABILITY AND ENERGY EFFICIENCY ANALYSIS OF EH-IWANC}

Here, the outage probability of the EH-IWANC protocol is obtained. Like it is depicted previously in Figure 2, the outage probability is $P_{o u t}=1-P_{s u c}$, where $P_{s u c}$ is the probability of successful transmission and it is mathematically expressed as

$$
P_{\text {suc }}=P_{\text {out }}^{\text {phase } 1}+P_{\text {out }}^{\text {phase } 3}
$$

$P_{\text {out }}^{\text {phase } 1}$ is the outage probability of the phase $1, P_{\text {out }}^{\text {phase } 3}$ is the outage probability of the phase 3 . We can mathematically express the phase 1 outage probability as

$$
P_{\text {outage }}^{\text {phase } 1}=1-\left(\overline{P_{\text {out }}^{h}}, \overline{P_{\text {out }}^{\grave{h}}}, \overline{P_{\text {out }}^{g}}, \overline{P_{\text {out }}^{\grave{g}}}\right)
$$

$\overline{P_{\text {out }}^{h}}, \overline{P_{\text {out }}^{\grave{h}}}, \overline{P_{\text {out }}^{g}}$, and $\overline{P_{\text {out }}^{\grave{g}}}$ are the successful transmission probability of the links $h, \grave{h}, g$ and $\grave{g}$, then, therefore they can express as

$$
\begin{aligned}
& \overline{P_{\text {out }}^{h}}=\operatorname{Pr}\left(I_{h}>\frac{R}{(1-\omega) / 2}\right)=\operatorname{Pr}\left(\log _{2}\left(1+P_{1}|h|^{2}\right)>\frac{R}{(1-\omega) / 2}\right)=\operatorname{Pr}\left(|h|^{2}>\frac{f_{h}}{P_{1} d_{h}^{-\alpha}}\right)=\exp \left(-\frac{f_{h}}{P_{1} d_{h}^{-\alpha}}\right), \\
& \overline{P_{\text {out }}^{\grave{h}}}=\operatorname{Pr}\left(I_{\grave{h}}>\frac{R}{(1-\omega) / 2}\right)=\operatorname{Pr}\left(\log _{2}\left(1+P_{1}|\grave{h}|^{2}\right)>\frac{R}{(1-\omega) / 2}\right)=\operatorname{Pr}\left(|\grave{h}|^{2}>\frac{f_{\grave{h}}}{P_{1} d_{\grave{h}}^{-\alpha}}\right)=\exp \left(-\frac{f_{\grave{h}}}{P_{1} d_{\grave{h}}^{-\alpha}}\right), \\
& \overline{P_{\text {out }}^{g}}=\operatorname{Pr}\left(I_{g}>\frac{R}{\omega / 2}\right)=\operatorname{Pr}\left(\log _{2}\left(1+P_{1}|g|^{2}\right)>\frac{R}{\omega / 2}\right)=\operatorname{Pr}\left(|g|^{2}>\frac{f_{g}}{P_{2}}\right)=\exp \left(-\frac{f_{g}}{P_{2} d_{g}^{-\alpha}}\right), \\
& \overline{P_{\text {out }}^{\grave{g}}}=\operatorname{Pr}\left(I_{\grave{g}}>\frac{R}{\omega / 2}\right)=\operatorname{Pr}\left(\log _{2}\left(1+P_{1}|\grave{g}|^{2}\right)>\frac{R}{\omega / 2}\right)=\operatorname{Pr}\left(|\grave{g}|^{2}>\frac{f_{\grave{g}}}{P_{2} d_{\grave{g}}^{-\alpha}}\right)=\exp \left(-\frac{f_{\grave{g}}}{P_{2} d_{\grave{g}}^{-\alpha}}\right) .
\end{aligned}
$$

where $f_{h}=2^{\frac{2 R}{(1-\omega)}}-1, f_{\grave{h}}=2^{\frac{2 R}{(1-\omega)}}-1, f_{g}=2^{\frac{2 R}{\omega}}-1$ and $f_{\grave{g}}=2^{\frac{2 R}{\omega}}-1$. Before we start analyzing phase 3 outage probability, let find transmission power of the phase 3 that is harvested from the phase 2 . The $P_{3}$ is a transmission power of the master node that harvested from the T2 device at phase 2 (time slot $\omega$ ). The energy harvested from the device of $\mathrm{T} 2$ can be shown like the following: 


$$
E_{k}=\frac{(1-\omega) T}{2} \varphi P_{3}|k|^{2}
$$

in which, $\varphi$ is the energy conversation ratio and it is vary between 0 to $1, P_{4}$ is the transmission power of $\mathrm{T} 2$ device. Thus, the transmission power of master node at phase 3 is given as

$$
P_{4}=\frac{E_{h}}{\omega T / 2}=\frac{1-\omega}{\omega} \varphi P_{3}|k|^{2}
$$

To this end, the outage probability of the phase 3 is given as

$$
\begin{aligned}
P_{\text {outage }}^{\text {phase }} & =1-\operatorname{Pr}\left(I_{\hat{f}}>\frac{R}{\frac{\omega}{2}}\right)=1-\operatorname{Pr}\left(\log _{2}\left(1+\frac{1-\omega}{\omega} \varphi P_{3}|k|^{2}|\hat{k}|^{2}\right)>\frac{R}{\omega / 2}\right) \\
& =1-\operatorname{Pr}\left(|k|^{2}|\hat{k}|^{2}>\frac{f_{\hat{f}}}{\bar{\omega}}\right)=1-\operatorname{Pr}\left(|k|^{2}|\hat{k}|^{2}>\bar{f}\right) \\
& =1-\int_{0}^{\infty} \frac{\int_{\bar{f}}^{\infty}}{|\bar{k}|^{2}} \frac{1}{d_{k}^{-\alpha}} \exp \left(\frac{|k|^{2}}{d_{k}^{-\alpha}}\right) \cdot \frac{1}{d_{\bar{k}}^{-\alpha}} \exp \left(\frac{|\hat{k}|^{2}}{d_{\bar{k}}^{-\alpha}}\right) d \hat{k} d k \\
& =1-\frac{1}{d_{\tilde{f}}^{-\alpha}} \sqrt{\frac{4 d_{\bar{k}}^{-\alpha} \bar{f}}{d_{k}^{-\alpha}}} K\left(\sqrt{\frac{4 \bar{f}}{d_{\bar{k}}^{-\alpha} d_{k}^{-\alpha}}}\right)
\end{aligned}
$$

where, $\bar{f}=\left(\left(2^{\frac{2 R}{\omega}}-1\right) \omega\right) /\left((1-\omega) \varphi P_{3}\right)$. Insert (3) in (2), then insert (2) in (1), and insert (6) in (1), we obtain the outage probability of the EH-IWANC protocol as

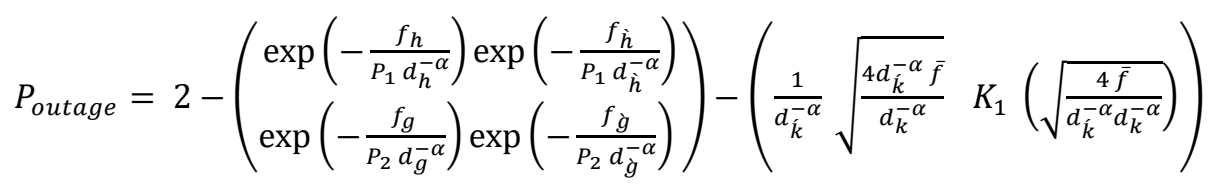

\section{SIMULATIONS AND RESULTS DISCUSSION}

The suggested Inter-WABN cooperating of every Internet of Medical Things system built on heath by predicting processes on PCs is inspected and sxamined over this section. We have considered the following assumption thorugh our simulation, a topolagy without method, every different over the air body sensor is put in a $3 \times 3 \mathrm{~m}$, as well as double body have been presumed co-exist within the same area of the transmission range. Every link is persumed to possess the identical distance amonge them, $d_{o}$. The ratio of transmitting of the every link have been persumed to be $\beta_{o}(b / s / H z)$. The path loss exponent, can be considered as $4, M l=40 \mathrm{~dB}$ and $N f=10 \mathrm{~dB}$, the total antenna gain is $G=5 \mathrm{dBi}$, the carrier frequency is $f c=2.5 \mathrm{GHz}$, and $N_{o}=-70 \mathrm{dBm}$. In the following, the power consuming of electric circuits for amplification is indicated, transmittion as well as reception like $P_{o}(\mathrm{~mW})$.

In the Figure 3, the outage probability like a function for internode distance is plotted. It is noticed that, the outage probability of the suggested protocol, eh-ANC, is less than eh-IW and eh-TH. Where, eh-IW is the energy harvesting over inter-WBAN cooperation without network coding, and eh-TH is energy harvesting over two-hop cooperation without network coding. In addition, the outage probability raised like the inter-node distance raised.

In the Figure 4, the outage probability like a function for assigned time slot has been plotted. It is noticed that, the outage probability for the suggested protocol is less matched with eh-ANC, eh-IW and eh-TH. Furthermore, the outage probability reduced as time for energy harvesting is more. In the Figure 5, the outage probability like a function for harvested power is plotted. It is noticed that, the outage probability for the suggested protocol is less matched with eh-IW and eh-TH. Besides, the outage probability reduced as the harvested power increased. 


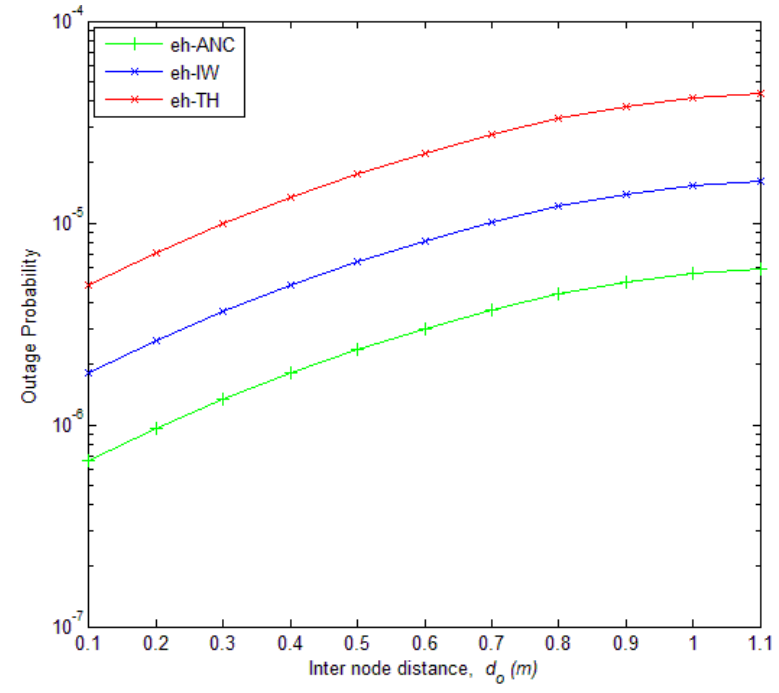

Figure 3. Outage probability versus internode distance

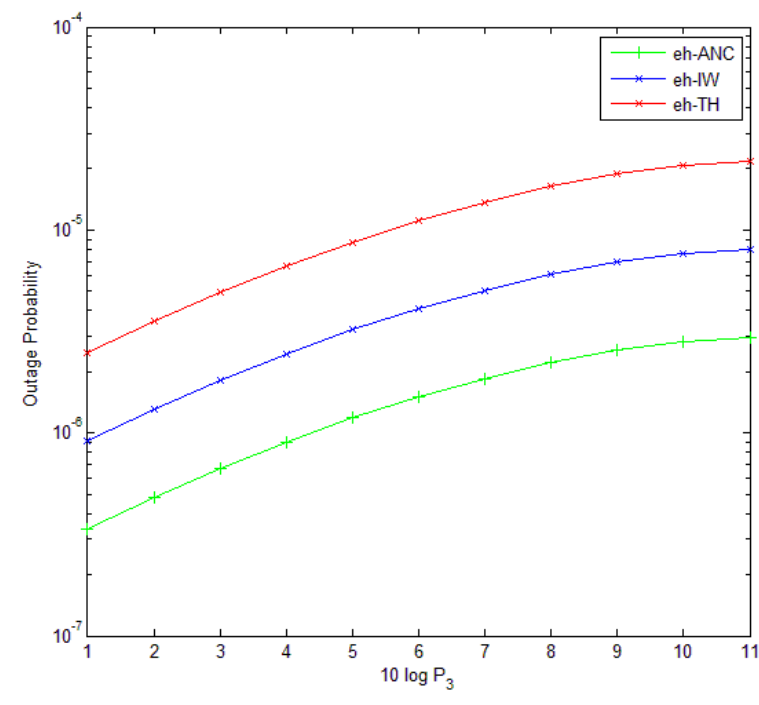

Figure 4. Outage probability versus harvested power

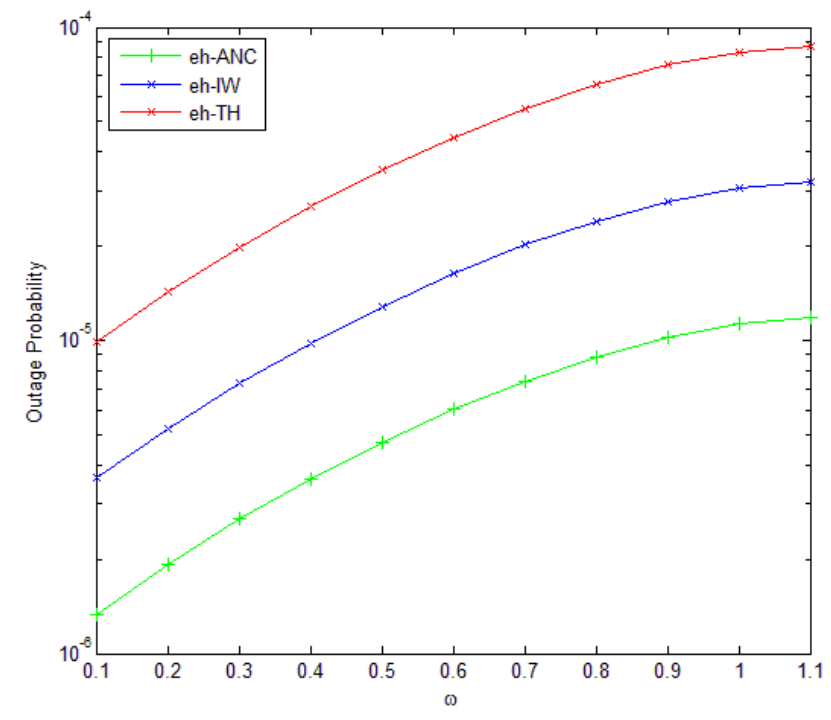

Figure 5. Outage probability versus time assignment factor

\section{CONCLUSION}

In this work, we proposed Energy Harvesting-based Inter-WBSN cooperation Network Coding (EH-IWCNC) for Internet of Medical Things. The outage probability of the EH-IoMT design has been studied theoretically with a specific term for the concluded outage over decode and forward (DF) energy harvesting scheme. We compared the proposed protocol with two different protocol, named as, eh-IW and eh-TH. The results shown that, the suggested protocol performed more superior if we compare it with the existing work.

\section{REFERENCES}

[1] G. Yang, et al., "A health-IoT platform based on the integration of intelligent packaging, unobtrusive bio-sensor, and intelligent medicine box," IEEE transactions on industrial informatics, vol. 10, no. 4, pp. 2180-2191, 2014.

[2] M. Hassanalieragh, et al., "Health monitoring and management using Internet-of-Things (IoT) sensing with cloudbased processing: Opportunities and challenges," in 2015 IEEE International Conference on Services Computing, pp. 285-292, 2015. 
[3] A. A. Thabit, et al., "Energy harvesting Internet of Things health-based paradigm: Towards outage probability reduction through inter-wireless body area network cooperation," International Journal of Distributed Sensor Networks, vol. 15, no. 10, pp. 1-12, 2019.

[4] D. A. Hammood, et al., "Body-to-Body Cooperation in Internet of Medical Things: Toward Energy Efficiency Improvement," Future internet, vol. 11, no. 11, p. 239-251, 2019.

[5] R. A. Khan and A. K. Pathan, "The state-of-the-art wireless body area sensor networks: A survey," International Journal of Distributed Sensor Networks, vol. 14, no. 4, pp. 1-23, 2018.

[6] H. Al-Mishmish, et al., "Critical Data-Based Incremental Cooperative Communication for Wireless Body Area Network," Sensors, vol. 18, pp. 3661-3678, 2018.

[7] D. A. Hammood, et al., "Reliable emergency data transmission using transmission mode selection in wireless body area network," Cogent Engineering, vol. 5, no. 1, pp. 198-206, 2018.

[8] D. Mishra, et al., "Smart RF energy harvesting communications: Challenges and opportunities," IEEE Communications Magazine, vol. 53, no. 4, pp. 70-78, 2015.

[9] L. R. Varshney, "Transporting information and energy simultaneously," in 2008 IEEE International Symposium Information Theory (ISIT 2008), pp. 1612-1616, 2008.

[10] L. Wang, et al., "Wireless information and power transfer to maximize information throughput in WBAN," IEEE Internet of Things Journal, vol. 4, no. 5, pp. 1663-1670, 2017.

[11] M. Ghamari, et al., "A survey on wireless body area networks for ehealthcare systems in residential environments," Sensors, vol. 16, no. 6, p. 831, 2016.

[12] Y. I. N. Yuehong, et al., "The internet of things in healthcare: An overview," Journal of Industrial Information Integration, vol. 1, pp. 3-13, 2016.

[13] K. Yeh, "A secure IoT-based healthcare system with body sensor networks," IEEE Access, vol. 4, pp. 10288-10299, 2016.

[14] R. M. Madhumathi, et al., "Healthcare Monitoring System Using Body Sensor Network," in International Conference on Engineering Innovations and Solutions (ICEIS-2016), pp. 171-176, 2016.

[15] S. R. Moosavi, et al., "End-to-end security scheme for mobility enabled healthcare Internet of Things," Future Generation Computer Systems, vol. 64, pp. 108-124, 2016.

[16] E. Luo, et al., "Privacy Protector: Privacy-Protected Patient Data Collection in IoT-Based Healthcare Systems," IEEE Communications Magazine, vol. 56, no. 2, pp. 163-168, 2018.

[17] H. Tao, et al., "Secured data collection with hardware-based ciphers for iot-based healthcare," IEEE Internet of Things Journal, vol. 6, no. 1, pp. 410-420, 2019.

[18] R. Hamza, et al., "A privacy-preserving cryptosystem for IoT E-healthcare," Information Sciences, vol. 527, pp. 493-510, 2019.

[19] M. Elhoseny, et al., "Secure medical data transmission model for IoT-based healthcare systems," IEEE Access, vol. 6, pp. 20596-20608, 2018.

[20] A. Alkhayyat, et al., "WBSN in IoT Health-Based Application: Toward Delay and Energy Consumption Minimization," Journal of Sensors, vol. 2019, pp. 1-14, 2019.

[21] D. A. Hammood, et al., "An energy-efficient optimization based scheme for low power devices in wireless body area networks," Journal of Computational and Theoretical Nanoscience, vol. 16, no. 7, pp. 2934-2940, 2019.

[22] D. A. Hammood, et al., "Enhancement of the Duty Cycle Cooperative Medium Access Control for Wireless Body Area Networks," in IEEE Access, vol. 7, pp. 3348-3359, 2019.

[23] A. Alkhayyat, et al., "The role of delay and connectivity in throughput reduction of cooperative decentralized wireless networks," Mathematical Problems in Engineering, vol. 2015, pp. 1-10, 2015.

[24] A. Alkhayyat, "Joint next-hop/relay selection for distributive multihop cooperative networks," Discrete Dynamics in Nature and Society, vol. 2015, pp. 1-10, 2015.

[25] A. Alkhayyat and S. B. Sadkhan, "Bandwidth efficiency analysis of cooperative communication with Reactive Relay Selection," 2018 International Conference on Engineering Technology and their Applications (IICETA), Al-Najaf, pp. 77-80, 2018.

[26] A. alkhayyat, et al., "Single Relay Selection in the Cognitive Cooperative Network: Toward Bandwidth Efficiency Improvement," 2019 4th Scientific International Conference Najaf (SICN), Al-Najef, Iraq, pp. 222-226, 2019.

[27] H. R. M. Al-Mishmish, et al., "Improvement of Underlay Cooperative Cognitive Networks Bandwidth Efficiency under Interference and Power Constraints," KSII Transactions on Internet and Information Systems (TIIS), vol. 13, no. 11 , pp. 5335-5353, 2019.

[28] A. Alkhayyat and N. A. Habeeb, "A Cooperative MAC Aware Network Coding toward Improving Throughput Wireless Body Area Network," 2019 2nd Scientific Conference of Computer Sciences (SCCS), Baghdad, Iraq, pp. 182-187, 2019.

[29] A. Alkhayyat and M. S. Mahmoud, "Hybrid Network Coding and Cooperative Communication in WBAN," 2019 2nd International Conference on Engineering Technology and its Applications (IICETA), Al-Najef, Iraq, pp. 79-82, 2019.

[30] A. Alkhayyat and M. S. Mahmoud, "Novel cooperative mac aware network coding under log-normal shadowing channel model in wireless body area network," International Journal on Communications Antenna and Propagation, vol. 9, no. 3, pp. 198-206, 2019. 


\section{BIOGRAPHIES OF AUTHORS}
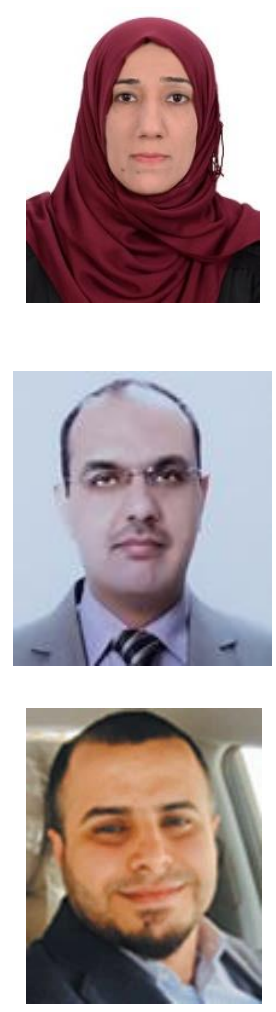

Dalal Abdulmohsin Hammood received the B.Sc. degree in Computer Engineering and Information Technology from the University of Technology- Baghdad, in 2003, and the M.Sc. in Computers Engineering Techniques from the Electrical Engineering Technical College, Middle Technical University, in 2011. She is currently pursuing the Ph.D, with the School of Computer and Communication Engineering, Universiti Malaysia Perlis, Perlis, Malaysia. Her research interests include computer networks security, Cryptography, Wireless Sensor Networks, Wireless Body Area Networks, and Image Processing, IoT. The algorithms that she works are: Artificial Intelligence such as, Artificial Neural Networks, Genetic Algorithms, PSO, Memetic Algorithm, and Optimization Algorithm.

Mahmoud Shuker Mahmoud received B.Sc. in Computer Engineering / Al-Nahrain University 1994-1997 and in Computer Engineering / Al-Nahrain University 1997-2000. He had Ph.D. in Computer Engineering / Al-Nahrain University 2003-2007. His research interest, IoT, Cyberscrity, routing protocol

Ahmed Alkhayyat received the B.Sc. degree in electrical engineering from AL KUFA University, Najaf, Iraq, in 2007, and the M.Sc. degree from the Dehradun Institute of Technology, Dehradun, India, in 2010. He contributed in organizing several IEEE conferences, workshop, and special sessions. He is currently a dean of international relationship and manager of the world ranking in the Islamic University, Najaf, Iraq. To serve his community, he acted as a reviewer for several journals and conferences. His research interests include network coding, cognitive radio, efficientenergy routing algorithms, and efficient-energy MAC protocol in cooperative wireless networks and wireless local area networks, WBAN, as well as cross-layer designing for self-organized network. 